\title{
Prevalence of Salmonella and Antimicrobial Resistance in Isolates from Food Animals - Six PLADs, China, 2019
}

\author{
Tingting Cao'; Peng Liu'; Yiming $\mathrm{Li}^{1}$; Mingquan Cui ${ }^{2}$; Chunping Zhang'; Yang Wang'; \\ Zhangqi Shen'; Jianzhong Shen'; Yuebin Ke ${ }^{3}$; Shaolin Wang ${ }^{1, * ;}$; Yongning $\mathrm{Wu}^{4, * *}$
}

\begin{abstract}
Summary
What is already known about this topic?

Salmonella causes acute and chronic diseases in food animals, and infected food animals are one of the most important source of human infection.

What does this report contribute?

The prevalence of Salmonella was $10.5 \%$ in chicken samples, $24.4 \%$ in pig, $23.3 \%$ in duck, and $29.4 \%$ in milk. Salmonella isolates were highly resistant to ampicillin (59.60\%).

What are the implications for public health practices?

Data on Salmonella infections among food animals in China could help identify sources and factors related to the spread of Salmonella in food animals and food production chains.
\end{abstract}

Salmonella bacterial infections have become a major public health issue, causing a wide range of clinical manifestations, including acute gastroenteritis and bacteremia. Antibiotics are commonly used to treat and control salmonellosis in food animals, contributing to the increasing prevalence of antibiotic-resistant Salmonella that has been attracting worldwide attention (1). Thus, investigating the prevalence of resistance-related genes in Salmonella could enhance the understanding of drug impacts on epidemiology. The study mainly followed the 2019 National Surveillance Program of Antibiotic Resistance in Bacteria of Food Animal Origins to conduct animalfood sampling in 6 provincial-level administrative divisions (PLADs) in China: Hebei, Shanxi, Shandong, Sichuan, Inner Mongolia, and Beijing. In addition to a minor decline in prevalence of Salmonella in chicken, results showed an increase in prevalence of Salmonella in pigs, ducks, and milk. Salmonella contamination of food animals has become a serious public health threat in China. Through antimicrobial susceptibility testing, Salmonella of animal origin was found to have multiple drug resistance and a high rate to ampicillin (59.6\%).
Therefore, in the animal breeding environment, public health practitioners should pay attention to the disinfection of the breeding farm environment and reduce the overuse of therapeutic drugs, promote the scientific use of drugs in the breeding process, and ensure the safety of public health.

In this study, a total of 1,493 non-duplicate samples were collected and stored in ESwabs (a swab-based collection kit) from animal farms and 85 from milk storage tanks on dairy farms. The sample collection method strictly complies with 2019 National Surveillance Program of Antibiotic Resistance in Bacteria of Food Animal Origins. Salmonella isolates were identified by matrix-assisted laser desorption ionization time-of-flight mass spectrometry (MALDITOF MS). Salmonella serotyping was performed using the White-Kauffmann-Le Minor scheme. Minimum inhibitory concentrations (MICs) were determined by the broth microdilution method according to the recommendations in the Clinical and Laboratory Standards Institute guidelines (CLSI, 2015: M100S25). Whole genome sequencing was conducted using an Illumina HiSeq2500 platform (Bionova Biotech Co. Beijing, China). Multilocus sequence typing (MLST) results were analyzed using MLST Version 2 (Seemann T, mlst Github http://github.com/ tseemann/mlst), and plasmid replicon typing was conducted using online tools (Center for Genomic Epidemiology, Technical University of Denmark, http://www.genomicepidemiology.org/). Resistance genes were identified using SRST2 Toolkit (version 0.2.0, The University of Melbourne, http://katholt. github.io/srst $2 /$ ).

A total of 198 Salmonella isolates (198/1,578, $12.6 \%$ ) were obtained from food animal samples from Hebei, Shanxi, Shandong, Sichuan, Inner Mongolia, and Beijing in 2019. Samples were collected from the feces, cecum, and milk of food animals. The highest rates of Salmonella isolates were obtained with the samples from cows. Beijing displayed the highest Salmonella isolation rate among the examined PLADs $(23.3 \%)$, while Hebei displayed the lowest isolation 
rate $(8.8 \%)$ (Table 1$)$.

Salmonella serotyping divided 133 isolates into 35 serotypes, with 65 isolates being incapable of being typed (Table 2). Salmonella Enteritidis (S. Enteritidis; $37.6 \%, 50 / 133)$ was the predominant species, followed by $S$. Tympanum $(9.0 \%, 12 / 133)$ and $S$. Kentucky (9.0\%, 12/133). Notably, multidrug resistant (MDR) strains were widely distributed among the various Salmonella serotypes. Among all the serotypes, $S$. Agona (77.8\%) showed the highest rates of antimicrobial resistance and MDR in the present study.

The MDR rates among Salmonella from animal sources were different in different PLADs. Beijing displayed the highest rate of MDR, reaching $100 \%$ (Figure 1A), followed by Shandong (52.0\%), Inner Mongolia (50.0\%), Sichuan (48.0\%), Shanxi (45.7\%), and Hebei (25.0\%). Salmonella isolates from six different PLADs were highly resistant to ampicillin (Figure 1B and 1C). A total of 18 strains of Salmonella with unique drug-resistant phenotypes were selected for whole-genome sequencing. Most Salmonella strains were resistant to multiple drugs. These strains were derived from chickens and pigs, and the MLST type was mainly ST11. The predominant serotype among these 18 Salmonella strains was enteritidis.

\section{DISCUSSION}

In the present study, the prevalence of Salmonella was $10.5 \%$ in chicken samples, $24.4 \%$ in pig, $23.3 \%$ in duck, and $29.4 \%$ in milk. The high contamination rate of Salmonella in milk samples indicated that milk is an important medium for Salmonella transmission. These results indicated that Salmonella contamination of food animals in China was a serious public health problem. Better measures should thus be taken to control Salmonella on dairy farms. Antimicrobial susceptibility tests in this study revealed that all the 198 Salmonella isolates were highly resistant to at least one tested antibiotic class (penicillin, folate pathway antagonists, tetracyclines, quinolones, and fluoroquinolones). The highest rates of antimicrobial resistance were observed for ampicillin (59.6\%). High prevalence of resistance to ampicillin is due to this antibiotic has been widely used in animal husbandry in China (2). At present, clinics have reported high resistance of Salmonella to ampicillin (53\%) (3) and high resistance in food animals $(68.7 \%)$ in China (4). We should carry out regular disinfection and sterilization of the breeding environment and avoid eating meat, eggs, and other dairy products that have not been treated with high temperatures. This research provided important guidance and reference value for animal breeding drugs, and provided data reference value for the detection, diagnosis, and treatment of Salmonella infection in clinic.

Compared with other research reports in China, the prevalence of Salmonella was $43.3 \%$ for chickens (5), $17.4 \%$ for pigs (O), $2.1 \%$ for ducks ( 7 ), and $1.3 \%$ for

TABLE 1. Characteristics and prevalence of Salmonella isolates from the 6 PLADs, China, 2019.

\begin{tabular}{ccccccc}
\hline PLAD & Sample & Source & No. of samples & No. of isolates & Isolating rate (\%) & 95\% Cl \\
\hline Shanxi & Cow & Milk & 85 & 25 & 29.4 & $(20.0,40.3)$ \\
& Chicken & Cecum & 121 & 10 & 8.3 & $(4.0,14.7)$ \\
Sichuan & Chicken & Cecum & 144 & 14 & 9.7 & $(5.4,15.8)$ \\
& & Fecal & 60 & 14 & 23.3 & $(13.4,36.0)$ \\
& Pig & Fecal & 90 & 22 & 24.4 & $(16.0,34.6)$ \\
Beijing & Duck & Fecal & 30 & 7 & 23.3 & $(9.9,42.3)$ \\
Shandong & Chicken & Cecum & 247 & 26 & 10.5 & $(7.0,15.0)$ \\
& & Fecal & 712 & 72 & 10.1 & $(8.0,12.6)$ \\
Inner Mongolia & Chicken & Cecum & 39 & 4 & 10.3 & $(2.9,24.2)$ \\
Hebei & Chicken & Fecal & 50 & 4 & 14.0 & $(2.2,19.2)$ \\
Total & Chicken & Cecum/fecal & 1,373 & 22 & 10.5 & $(8.9,12.2)$ \\
& Pig & Fecal & 90 & 7 & 24.4 & $(16.0,34.6)$ \\
& Duck & Fecal & 30 & 25 & 23.3 & $(9.9,42.3)$ \\
& Cow & Milk & 85 & 29.4 & $(20.0,40.3)$ \\
\hline
\end{tabular}

Note: The six PLADs inculdes Hebei, Shanxi, Shandong, Sichuan, Inner Mongolia, and Beijing. The primary objective of the present study was to investigate the Isolating Rate and corresponding 95\% Cls of prevalence of Salmonella isolates from the 6 PLADs, China, in 2019. Abbreviations: PLADs=provincial-level administrative divisions; $\mathrm{Cl}=$ confidence interval. 
TABLE 2. Salmonella serovar isolates obtained from chicken, pig, cow, and duck samples from the six PLADs, China, 2019.

\begin{tabular}{|c|c|c|c|c|c|}
\hline Serovar & Chicken & Pig & Cow & Duck & Total \\
\hline Enteritidis & 44 & 5 & & 1 & 50 \\
\hline Tymphimurium & 1 & 6 & 5 & & 12 \\
\hline Kentucky & 12 & & & & 12 \\
\hline Agona & 4 & 2 & 3 & & 9 \\
\hline Djugu & 6 & & & & 6 \\
\hline Corvallis & 4 & & & & 4 \\
\hline Paratyphi B & 4 & & & & 4 \\
\hline Essen & 2 & 1 & & & 3 \\
\hline Koenigstuhl & 1 & & & 1 & 2 \\
\hline Norwich & 1 & 1 & & & 2 \\
\hline Schwarzengrund & & 2 & & & 2 \\
\hline Trachau & 2 & & & & 2 \\
\hline Meleagridis & & & 2 & & 2 \\
\hline Kedougou & & 2 & & & 2 \\
\hline Kingston & 1 & & & & 1 \\
\hline Shannon & 1 & & & & 1 \\
\hline Stuttgart & 1 & & & & 1 \\
\hline Bovismorbificans & 1 & & & & 1 \\
\hline Mbandaka & 1 & & & & 1 \\
\hline Uppsale & & 1 & & & 1 \\
\hline Eschweiler & 1 & & & & 1 \\
\hline Braenderup & 1 & & & & 1 \\
\hline Derby & & 1 & & & 1 \\
\hline Havana & & 1 & & & 1 \\
\hline Anatum & & 1 & & & 1 \\
\hline Waycross & & 1 & & & 1 \\
\hline Bareilly & & 1 & & & 1 \\
\hline Azteca & & 1 & & & 1 \\
\hline Gueuletapee & 1 & & & & 1 \\
\hline Bloomsbury & 1 & & & & 1 \\
\hline Hato & & & & 1 & 1 \\
\hline Powel 2 & 1 & & & & 1 \\
\hline Rissen & & 1 & & & 1 \\
\hline Menden & 1 & & & & 1 \\
\hline Nola & 1 & & & & 1 \\
\hline Total & 93 & 27 & 10 & 3 & 133 \\
\hline
\end{tabular}

Note: The six PLADs inculdes Hebei, Shanxi, Shandong, Sichuan, Inner Mongolia, and Beijing.

Abbreviation: PLADs=provincial-level administrative divisions.

milk (8). In addition to the reduction in the prevalence of Salmonella from chickens, our results showed an increase in the prevalence of Salmonella in pigs, ducks, and milk. In this study, the high rate of Salmonella contamination in cow milk samples. Other studies have reported Salmonella infection in raw milk and milk-related infant foods (9) as well, which might threaten the health of babies. Although the milk sample sources used in this study were limited, the findings suggested that Salmonella poses a threat to the milk supply. The highest rates of antimicrobial resistance were observed for ampicillin (59.6\%), in agreement with the results of many previous studies on Salmonella isolates from food animals (10).

This study was subject to several limitations. First, the geographically concentrated nature of the samples in the present study does not represent China as a whole. Second, the types of the samples collected from each of the six PLADs and cities were not uniform, leading to sample biases as the collected samples could not represent the overall circumstances.

This was a survey of the prevalence of Salmonella in samples obtained from food animals from six PLADs in China. The isolates showed high antimicrobial resistance, with resistance to ampicillin being the most common. It is worth noting that in this study, $S$. Enteritidis displayed the most prevalent drug resistance and MDR. MDR Salmonella isolates from humans have a common ancestor with the isolates from food animals, increasing the difficulty of curing human infections and increasing healthcare costs. A nationally coordinated intervention strategy for drug use in farmed animals is needed to limit the spread of MDR Salmonella. Better methods for monitoring the emergence and spread of MDR Salmonella would facilitate disease control and treatment. To prevent these strains from becoming a worldwide pandemic, internationally coordinated intervention strategies to limit further dissemination of MDR Salmonella are required.

Acknowledgement: Research staff at College of Veterinary Medicine, China Agricultural University.

Conflicts of interest: No conflicts of interest.

Funding: The National Key Research and Development Program of China (2016YFD0501301) and National Science Foundation of China (31761133004 and 81861138051).

doi: $10.46234 / \mathrm{ccdcw} 2021.132$

\# Corresponding authors: Shaolin Wang, shaolinwang@cau.edu.cn; Yongning Wu, wuyongning@cfsa.cn.

\footnotetext{
College of Veterinary Medicine, China Agricultural University, Beijing, China; ${ }^{2}$ China Institute of Veterinary Drug Control, Beijing, China; ${ }^{3}$ Key Laboratory of Molecular Epidemiology of Shenzhen, Shenzhen Center for Disease Control and Prevention, Shenzhen, Guangdong, China; ${ }^{4}$ National Health Commission Key Laboratory of Food Safety Risk Assessment, Food Safety Research Unit
} 


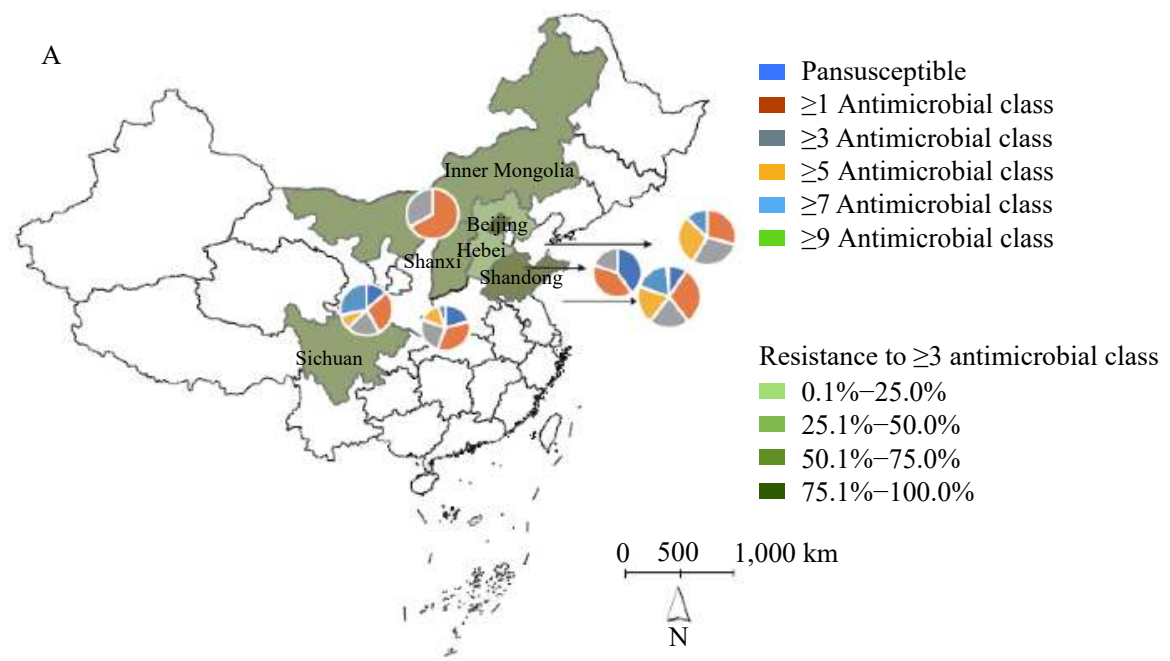

B

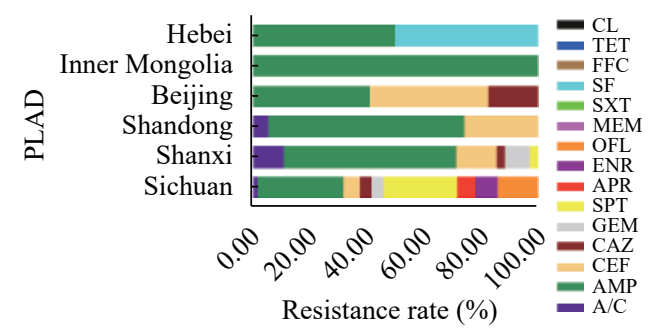

$c$

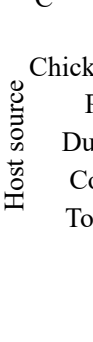

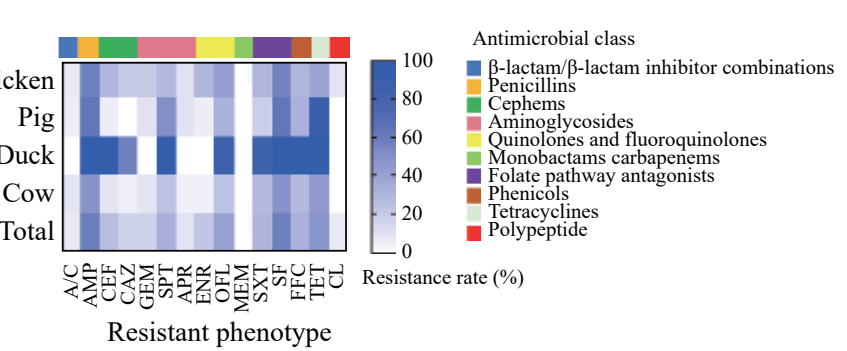

FIGURE 1. Multidrug resistance and distribution of Salmonella from the six PLADs, China, 2019. (A) The proportion of different antibiotic resistant types. (B) Distribution of Salmonella resistance phenotypes from different PLADs and cities in China. (C) Resistant phenotypes of Salmonella from different host sources.

Note: The six PLADs include Hebei, Shanxi, Shandong, Sichuan, Inner Mongolia, and Beijing.

Abbreviations: PLADs=provincial-level administrative divisions; $A / C=$ amoxicilin-clavulanic acid; $A M P=a m p i c i l l i n ;$ $\mathrm{CEF}=$ ceftiofur; $\mathrm{CAZ}=$ ceftazidime; $\mathrm{GEM}=$ gentamicin; SPT=spectinomycin; $\mathrm{APR}=$ apramycin; $\mathrm{ENR}=$ enrofloxacin; $\mathrm{OFL}=$ ofloxacin; MEM=meropenem; SXT=trimethoprim-sulfamethoxazole; SF=sulfamethoxazole; FFC=florfenicol; TET= tetracycline; $\mathrm{CL}=$ colistin.

(2019RU014) of Chinese Academy of Medical Science, China National Center for Food Safety Risk Assessment, Beijing, China.

Submitted: March 06, 2021; Accepted: June 09, 2021

\section{REFERENCES}

1. Vinueza-Burgos C, Baquero M, Medina J, De Zutter L. Occurrence, genotypes and antimicrobial susceptibility of Salmonella collected from the broiler production chain within an integrated poultry company. Int J Food Microbiol 2019;299:1 - 7. http://dx.doi.org/10.1016/j.ijfood micro.2019.03.014.

2. Zhang QQ, Ying GG, Pan CG, Liu YS, Zhao JL. Comprehensive evaluation of antibiotics emission and fate in the river basins of China: source analysis, multimedia modeling, and linkage to bacterial resistance.EnvironSciTechnol2015;49(11):6772 - 82.http://dx.doi.org/ 10.1021/acs.est.5b00729.

3. Lv D, Zhang D, Song Q. Expansion of Salmonella Typhi clonal lineages with ampicillin resistance and reduced ciprofloxacin susceptibility in eastern China. Infect Drug Resist 2019;12:2215 - 21. http://dx.doi.org/ 10.2147/IDR.S208251.

4. Zhao XN, Hu M, Zhang Q, Zhao C, Zhang Y, Li LL, et al. Characterization of integrons and antimicrobial resistance in Salmonella from broilers in Shandong, China. Poult Sci 2020;99(12):7046 - 54. http://dx.doi.org/10.1016/j.psj.2020.09.071.

5. Yang BW, Cui Y, Shi C, Wang JQ, Xia XD, Xi ML, et al. Counts, serotypes, and antimicrobial resistance of Salmonella isolates on retail raw poultry in the People's Republic of China. J Food Prot 2014;77(6):894 - 902. http://dx.doi.org/10.4315/0362-028X.JFP-13439.

6. Li Q, Yin J, Li Z, Li ZW, Du YZ, Guo WW, et al. Serotype distribution, antimicrobial susceptibility, antimicrobial resistance genes and virulence genes of Salmonella isolated from a pig slaughterhouse in Yangzhou, China. AMB Express 2019;9(1):210. http://dx.doi.org/ 10.1186/s13568-019-0936-9.

7. Yang J, Ju ZJ, Yang Y, Zhao XN, Jiang ZY, Sun SH. Serotype, antimicrobial susceptibility and genotype profiles of Salmonella isolated from duck farms and a slaughterhouse in Shandong province, China. BMC Microbiol 2019;19(1):202. http://dx.doi.org/10.1186/s12866019-1570-z.

8. Lan XY, Zhao SG, Zheng N, Li SL, Zhang YD, Liu HM, et al. Short communication: Microbiological quality of raw cow milk and its association with herd management practices in northern China. J Dairy Sci 2017;100(6):4294 - 9. http://dx.doi.org/10.3168/jds.2016-11631.

9. Yang B, Zhao H, Cui S, Wang Y, Xia X, Xi M, et al. Prevalence and characterization of Salmonella enterica in dried milk-related infant foods in Shaanxi, China. J Dairy Sci 2014;97(11):6754-60. http://dx. doi.org/10.3168/jds.2014-8292.

10. Varga C, Guerin MT, Brash ML, Slavic D, Boerlin P, Susta L. Antimicrobial resistance in fecal Escherichia coli and Salmonella enterica isolates: a two-year prospective study of small poultry flocks in Ontario, Canada. BMC Vet Res 2019;15(1):464. http://dx.doi.org/10.1186/ s12917-019-2187-z. 